\title{
COVID-19 Impact on Colorectal Daily Practice-How Long Will It Take to Catch Up?
}

\author{
Dong Hum Yoon ${ }^{1} \cdot$ Sarah Koller ${ }^{1} \cdot$ Philip Marjun N. Duldulao ${ }^{1} \cdot$ Glenn T. Ault $^{1} \cdot$ Sang W. Lee ${ }^{1} \cdot$ Kyle G. Cologne $^{1,2} \mathbb{E}_{0}$
}

Received: 13 May 2020 / Accepted: 28 June 2020 / Published online: 27 July 2020

(C) 2020 The Society for Surgery of the Alimentary Tract

\section{Background}

All elective surgeries have been postponed at our institution starting 3/16/20 due to the COVID-19 pandemic. We assessed changes in hospital resource utilization and estimated the future backlog of cases in the colorectal surgery division of a large safety-net hospital.

\section{Methods}

Patients undergoing colorectal procedures from 3/16/20 to 4/23/20 (COVID) were compared with those from January through June 2018 (historical). Resource utilization rates were calculated by weekly case volumes and hospital stay in each group. A future catch up timeframe and new wait times from scheduling to surgery dates were calculated.

\section{Results}

The COVID and historical groups included 13 and 239 patients, respectively. The COVID group showed a 74\% relative decrease in weekly surgical case rates (9.2 to 2.4 patients per week). Both groups had similar lengths of stay. The COVID group had a longer average ICU stay $(1.4 \pm 2.5$ days vs. $0.4 \pm 1.2$ days, $P=0.016)$ and a $132 \%$ increase in ICU resource utilization. Overall, the COVID group had a $48 \%$ relative decrease in hospital resource utilization, owing to reduced volume but higher acuity. If the surgery numbers returns to pre-COVID volumes, the calculated "catch up" times range from 4.6 weeks to 9.2 weeks. Wait times for new cases may increase by $70 \%$ compared with pre-COVID levels.

\section{Conclusion}

Cancelling elective colorectal surgeries results in a decrease in overall but increase in ICU-specific resource utilization. Though necessary, cancellations result in an increasing backlog of cases that poses significant future logistical and clinical challenges in an already overburdened safety-net hospital. Effective triage systems will be critical to prioritize this backlog.

Keywords COVID-19 $\cdot$ Colorectal surgery $\cdot$ Resource utilization $\cdot$ Backlog

\section{Introduction}

In late December of 2019, a cluster of cases involving patients with interstitial pneumonia of unknown etiology emerged from the Hubei province of China. The virus was soon identified as a novel coronavirus and named SARS-CoV-2 by the Coronavirus Study Group ${ }^{1}$ with its associated disease labeled

Kyle G. Cologne

Kyle.Cologne@med.usc.edu

1 Division of Colorectal Surgery, Department of Surgery, LAC+USC, Los Angeles, CA, USA

21441 Eastlake Ave, Suite NTT-7418, Los Angeles, CA 90033-4612, USA as COVID-19 by the WHO. ${ }^{2}$ The virus spread rapidly so that by the end of January 2020, close to eight thousand cases were confirmed in China alone, and cases were seen in 18 other countries, including the USA. ${ }^{3}$ COVID-19 was deemed a Public Health Emergency of International Concern by the WHO at that time and subsequently as a pandemic on March 11, 2020, as the virus caused a global surge in cases. ${ }^{4}$

Following this announcement, national health groups of the USA published guidelines to restructure medical systems for reallocation of resources in anticipation of a surge of these novel respiratory virus cases. In particular, both the Centers for Disease Control and Prevention (CDC) and the American College of Surgeons recommended drastically reducing surgical case volumes by cancelling elective procedures and redirecting both personnel and equipment resources for anticipated COVID-19 patients. ${ }^{5,6}$ 
Our institution mandated cancellation of all elective procedures as of March 16 in accordance with the national guidelines. The immediate and long-term effects of this policy implementation are not known. This study aimed to quantify the change in hospital resource utilization after the policy implementation and to estimate the future burden of postponed surgeries in colorectal surgery.

\section{Methods}

\section{Design}

This is a retrospective study at a large urban safety-net hospital comparing a historical cohort of all patients over 18 years old who underwent elective or non-elective colorectal procedures from January through June of 2018 to those during the COVID-19 pandemic (March 16, 2020, to April 23, 2020). The historical cohort included 239 patients over 25.7 weeks (9.2 patients per week), while the pandemic group (COVID group) included 13 patients over 5.4 weeks (2.4 patients per week). The COVID group consisted of patients who were treated after the policy implementation on March 16, 2020, which allowed colorectal procedures for only non-elective or cancer cases. Non-elective cases included those that were either urgent or emergent, requiring an operation following an unplanned admission or within 12 hours, respectively. The historical control represented the usual hospital resource utilization pattern. A prospectively maintained, departmental database was used to identify all surgical cases. Endoscopic procedures were excluded.

Data regarding patient demographics, procedure types and indications, hospital resource utilization factors - including length of stay (LOS), intensive care unit (ICU) utilization, and emergency department (ED) visits - and 30-day postoperative morbidity and mortality were collected from the departmental database. Morbidity was graded by the Clavien-Dindo classification and also categorized by individual complications.

All cancelled or postponed elective colorectal cases following institutional policy implementation were characterized by procedure type, surgical indication, and inpatient admission requirement.

The primary outcomes were procedure volume, overall hospital resource utilization rates, and ICU utilization rates across the two periods. Secondary outcomes included LOS, ICU stay, 30-day post-discharge ED visits, unplanned readmissions/reoperations, and 30-day postoperative morbidity and mortality.

\section{Calculations and Statistics}

The average cases performed weekly, stratified by urgency and inpatient admission requirement, were calculated by dividing the total number of cases performed during each time interval by the number of weeks. Overall resource utilization was calculated by multiplying the average number of surgeries requiring admission per week by average LOS; this represented the number of patient days required to treat patients per week. ICU resource utilization was calculated similarly, except with average ICU stay. Cases not requiring inpatient admission were excluded from the above calculations.

In estimating the future burden of cases, we applied some assumptions. The total number of cases cancelled or postponed during the COVID period was tallied. The backlog of cases by May 31, 2020, was a sum of these cancelled cases plus the presumed number of newly scheduled elective cases (new case requests), minus any cases performed during the pandemic period. March 16 to May 31, 2020, represents twice the time length as the COVID group timeframe (76 and 38 days, respectively). Estimated "catch up" time intervals were calculated based on the lower weekly new case requests during the COVID timeframe as well as historical numbers of average weekly cases performed to return the patient backlog to baseline (March 16). New wait times were calculated based on 2-, 4-, and 6-week durations of predicted higher-thanhistorical new case requests before returning to historical numbers of new case requests. Wait times were defined as the time period between surgery scheduling and day of surgery.

Continuous parametric variables were compared using a two-tailed Student's $t$ test. Nonparametric nominal data were compared using a chi-squared test and Fisher's exact test, as appropriate, while nonparametric ordinal data were compared using a Mann-Whitney U test. Calculations were performed on Social Science Statistics online calculator and Microsoft Excel 2013. A $P$ value of less than 0.05 was considered significant.

\section{Results}

\section{Demographic and Surgical Data Comparison}

The historical group included 239 patients over a 25.7-week period with corresponding 9.3 cases performed per week, and the COVID groups included 13 patients over a 5.4-week period with corresponding 2.4 cases performed per week. There were no significant differences in age, sex, or body mass index (BMI) between groups. The COVID group had more patients with higher ASA classification (Table 1). The proportions of each procedure type performed were similar across both groups, with the exception of anorectal procedures, which showed an $87 \%$ reduction in the COVID group (8\% vs. $62 \%, P<0.001)$. When considering abdominal procedures, rates of open and minimally invasive approaches were similar. The only indication for elective procedures in the COVID 
group was cancer. There were no differences in indications for non-elective cases between the two groups.

The COVID group showed a $74 \%$ decrease in overall weekly surgical case volume relative to the historical group (2.4 cases/week vs. 9.3 cases/week, respectively) (Table 1). The COVID group had a much lower percentage of elective cases $(31 \%$ vs. $82 \%, P<0.001)$ compared with the historical group. The ratios of elective to non-elective cases were 1:2.3 and 1:0.2 for the COVID and historical groups, respectively. The COVID group also had a higher percentage of elective cases requiring inpatient admission $(100 \%$ vs. $34 \%, P=$ $0.014)$.

\section{Resource Utilization Outcomes}

The average LOS was similar between the COVID and historical groups $(9.0 \pm 6.7$ days vs. $10 \pm 21.6$ days, respectively, $P=0.882$ ) (Table 2). The COVID group had longer ICU stays $(1.4 \pm 2.5$ days vs. $0.4 \pm 1.2$ days, $P=0.016)$. The COVID group had $48 \%$ lower overall hospital resource utilization (21.6 patient-days/week vs. 41.5 patient-days/week) but $132 \%$ higher ICU resource utilization (3.4 patient-ICU days/ week vs. 1.5 patient-ICU days/week) compared with the historical group. The rates of ED visits, unplanned readmissions, and reoperations were similar, which remained unchanged after stratifying by procedure type (anorectal vs. abdominal).

\section{Morbidity Outcomes}

The overall postoperative complication rate was higher in the COVID group ( $46 \%$ vs. $20 \%, P=0.037$ ) (Table 3 ). When complications were stratified by Clavien-Dindo classification, there were no statistically significant differences between the two groups. The rates of specific complications were also similar between the two groups. In particular, there were no differences in surgical site infection or anastomotic leak rates. Though the historical group had higher rates of "other" complications, which included mesh infection, enterocutaneous fistula, and ostomy outlet obstruction, and the COVID group had higher DVT/PE rates, none were statistically significant. When excluding outpatient anorectal cases from the historical group, there was no difference in overall complication rate. There was no 30-day mortality in either group.

\section{Case Backlog Characteristics}

On March 16, 2020, there were 73 cases already scheduled, equating to a baseline wait time of 9.6 weeks (from the surgery scheduling to day of surgery). This was similar to the average historical backlog of cases and associated wait time from the pre-COVID period. Following the institutional policy implementation, 69 of these cases (95\%) were indefinitely postponed. None of these postponed cases were rescheduled to within the COVID cohort.

Examining the cases by procedure type, 4 cases $(5 \%)$ proceeded as elective, scheduled cases for oncologic reasons; 3 patients underwent resection for rectal (2) and sigmoid (1) cancers; and 1 patient underwent a scheduled loop sigmoid colostomy for metastatic rectal cancer with impending obstruction. Forty-seven $(68 \%)$ of cancelled procedures were outpatient anorectal cases (Table 4). The remaining 22 $(32 \%)$ were scheduled inpatient cases for benign disease processes, except for 3 patients: 2 with suspicious rectal and colon polyps who required advanced endoscopic surgical techniques, and 1 with rectosigmoid cancer who eventually transferred their care to another institution. Among the elective inpatient cases cancelled, the most common procedure type was an ostomy-related procedure (37\%).

\section{Estimates of Future Burden}

During the 25.7 weeks of the historical group, 196 elective (both inpatient and outpatient) procedures were performed (7.6 cases per week). This is in contrast to the 4 elective cases that were performed during the 5.4 weeks of the COVID group ( 0.7 cases per week), all of which were inpatient cancer cases. The elective inpatient case rates of the historical and COVID groups were 2.6 vs. 0.7 cases per week, respectively, and the elective outpatient anorectal case rates were 5.1 vs. 0 cases per week, respectively.

The non-elective case rates were comparable with both groups averaging 1.7 cases per week. Indications in the COVID group included perforated bowel due to Crohn's disease, anastomotic leak from a prior (non-COVID period) colon resection, large bowel obstruction from obstructing colorectal cancer, massive rectal bleeding due to a rectal Dieulafoy lesion, and acute diverticulitis failing conservative management (Table 1). Given the equivalent non-elective weekly case volume between the two groups, changes in only elective case volume were taken into account for the calculations below.

During the COVID group timeframe, 15 additional cases were added to the surgery scheduling backlog from clinic or inpatient non-urgent consults, for an average of 2.8 new case requests per week. Assuming the inflow and outflow of operative cases to be consistent during the pandemic, the backlog list is expected to grow by $30 \%$ to 95.2 cases (from 73 cases at baseline) by May 31 st. Assuming that the operative capacity of the hospital returns to historical numbers at that time, several scenarios are possible depending on the changes in new case requests. If the new case requests remain consistently low (2.8 new cases per week), 4.6 weeks would be needed to return to the baseline backlog of 73 patients (Fig. 1). If new case requests rise to an average somewhere between the pandemic and historical new case requests, it would take 9.2 weeks to return to the pre-COVID baseline backlog. 
Table 1 Characteristics of groups and surgery volumes

\begin{tabular}{|c|c|c|c|c|c|c|}
\hline & & \multicolumn{2}{|c|}{ Historical $(n=239)$} & \multicolumn{2}{|c|}{$\operatorname{COVID}(n=13)$} & \multirow[t]{2}{*}{$P$ value } \\
\hline & & $N$ & $\%$ & $\mathrm{~N}$ & $\%$ & \\
\hline \multicolumn{2}{|l|}{$\mathrm{Age}^{\mathrm{a}}$} & 48.5 & 13.0 & 55.6 & 10.0 & 0.052 \\
\hline \multirow[t]{2}{*}{ Sex } & Male & 154 & $64 \%$ & 10 & $77 \%$ & 0.396 \\
\hline & Female & 85 & $36 \%$ & 3 & $23 \%$ & \\
\hline \multicolumn{2}{|l|}{$\mathrm{BMI}^{\mathrm{a}, \mathrm{b}}$} & 28.4 & 6.0 & 38.0 & 8.4 & 0.837 \\
\hline \multirow[t]{4}{*}{ ASA } & I & 38 & $16 \%$ & 0 & $0 \%$ & 0.227 \\
\hline & II & 150 & $63 \%$ & 1 & $8 \%$ & $<0.001$ \\
\hline & III & 50 & $21 \%$ & 9 & $69 \%$ & $<0.001$ \\
\hline & IV & 1 & $0 \%$ & 3 & $23 \%$ & $<0.001$ \\
\hline \multicolumn{7}{|l|}{ Procedure types } \\
\hline & Anorectal & 147 & $62 \%$ & 1 & $8 \%$ & $<0.001$ \\
\hline & Segmental colectomy & 35 & $15 \%$ & 4 & $31 \%$ & 0.229 \\
\hline & Subtotal/total colectomy & 1 & $0 \%$ & 1 & $8 \%$ & 0.101 \\
\hline & LAR & 12 & $5 \%$ & 2 & $15 \%$ & 0.157 \\
\hline & APR & 0 & $0 \%$ & 0 & $0 \%$ & - \\
\hline & Ostomy creation & 13 & $5 \%$ & 2 & $15 \%$ & 0.176 \\
\hline & Ostomy reversal & 14 & $6 \%$ & 0 & $0 \%$ & 0.624 \\
\hline & Others ${ }^{\mathrm{c}}$ & 17 & $7 \%$ & 3 & $23 \%$ & 0.073 \\
\hline \multicolumn{7}{|l|}{ Surgical approach } \\
\hline & Open & 53 & $58 \%$ & 8 & $67 \%$ & 0.757 \\
\hline & Open conversion ${ }^{\mathrm{d}}$ & 7 & $8 \%$ & 0 & $0 \%$ & 0.596 \\
\hline & Laparoscopic & 19 & $21 \%$ & 4 & $33 \%$ & 0.459 \\
\hline & Robotic & 12 & $13 \%$ & 0 & $0 \%$ & 0.351 \\
\hline \multicolumn{7}{|c|}{ Elective case indications } \\
\hline & Anorectal & 129 & $66 \%$ & 0 & - & - \\
\hline & Cancer/mass & 36 & $18 \%$ & 4 & $100 \%$ & 0.001 \\
\hline & IBD & 2 & $1 \%$ & 0 & - & - \\
\hline & Diverticulitis & 7 & $4 \%$ & 0 & - & - \\
\hline & Ostomy related & 18 & $9 \%$ & 0 & - & - \\
\hline & Others $^{\text {e }}$ & 4 & $2 \%$ & 0 & - & - \\
\hline \multicolumn{7}{|c|}{ Non-elective case indications } \\
\hline & Anorectal emergencies ${ }^{\mathrm{f}}$ & 12 & $28 \%$ & 1 & $11 \%$ & 0.420 \\
\hline & Bowel perforation & 3 & $7 \%$ & 1 & $11 \%$ & 1.0 \\
\hline & Bowel obstruction & 9 & $21 \%$ & 4 & $44 \%$ & 0.203 \\
\hline & Diverticulitis & 5 & $12 \%$ & 1 & $11 \%$ & 1.0 \\
\hline & IBD complications & 1 & $2 \%$ & 0 & $0 \%$ & 1.0 \\
\hline & Others $^{\mathrm{g}}$ & 13 & $30 \%$ & 2 & $22 \%$ & 0.712 \\
\hline & & $\mathrm{N}(\%)$ & Rate $^{\text {h }}$ & $\mathrm{N}(\%)$ & Rate $^{\mathrm{h}}$ & $P$ value \\
\hline \multirow[t]{3}{*}{ Case rates } & Elective & $196(82)$ & 7.62 & $4(31)$ & 0.74 & $<0.001$ \\
\hline & Non-elective & $43(18)$ & 1.67 & $9(69)$ & 1.66 & \\
\hline & Overall & $239(100)$ & 9.29 & $13(100)$ & 2.40 & \\
\hline \multirow[t]{2}{*}{ Elective case rates } & Requiring admission & $66(34)$ & 2.57 & $4(100)$ & 0.74 & 0.014 \\
\hline & Outpatient anorectal & $130(66)$ & 5.06 & $0(0)$ & - & \\
\hline \multicolumn{7}{|c|}{ Non-elective case rates } \\
\hline & Requiring admission & $41(95)$ & 1.59 & $9(100)$ & 1.66 & 1.0 \\
\hline & outpatient anorectal & $2(5)$ & 0.08 & $0(0)$ & - & \\
\hline \multicolumn{7}{|l|}{ Requiring Admission } \\
\hline & & $107(45)$ & 4.16 & $13(100)$ & 2.39 & $<0.001$ \\
\hline
\end{tabular}

$B M I$ body mass index, ASA American Society of Anesthesiologists physical classification system, LAR low anterior resection, $A P R$ abdominoperineal resection, $I B D$ inflammatory bowel disease

${ }^{\mathrm{a}}$ Results in mean and standard deviation

${ }^{\mathrm{b}}$ Two patients from historical group had no recorded BMI

${ }^{\mathrm{c}}$ Includes appendectomy, ileal pouch-anal anastomosis, ileal pouch excision, pelvic exenteration, enterocutaneous fistula takedown, radical resection of retroperitoneal tumor, peristomal abscess incision and drainage, TAMIS, small bowel resection, ileostomy revision, and enterocolonic bypass; the last three consisted "other" procedures in the COVID group

${ }^{\mathrm{d}}$ Converted from either initially laparoscopic or robotic approach

${ }^{\mathrm{e}}$ Includes chronic appendicitis, large bowel obstruction, colonic stricture, and rectovaginal fistula

${ }^{\mathrm{f}}$ Abscess, fistula, thrombosed/incarcerated hemorrhoid, rectal bleeding

${ }^{\mathrm{g}}$ Includes sigmoid volvulus, anastomotic leak, intraperitoneal bleeding, fecal diversion for sacral decubitus ulcer, and parastomal abscess.

${ }^{\mathrm{h}}$ Number of cases per week 
Table 2 Comparisons of resource utilization overall and excluding outpatient anorectal cases

\begin{tabular}{|c|c|c|c|c|c|c|c|c|}
\hline & \multicolumn{2}{|c|}{ Historical overall $(n=239)$} & \multicolumn{2}{|c|}{ Historical minus OAR $(n=112)^{\mathrm{a}}$} & \multicolumn{2}{|c|}{$\operatorname{COVID}(n=13)$} & \multirow[t]{2}{*}{$P$ value ${ }^{\mathrm{b}}$} & \multirow[t]{2}{*}{$P$ value } \\
\hline & Avg & SD & Avg & $\mathrm{SD}$ & Avg & $\mathrm{SD}$ & & \\
\hline LOS & 10.0 & 21.6 & 10.0 & 21.6 & 9.0 & 6.7 & 0.882 & 0.882 \\
\hline ICU & 0.4 & 1.2 & 0.4 & 1.2 & 1.4 & 2.5 & 0.016 & 0.016 \\
\hline Overall utilization $^{\mathrm{d}}$ & 41.5 & - & 41.5 & - & 21.6 & - & & \\
\hline \multirow[t]{2}{*}{ ICU utilization ${ }^{\mathrm{d}}$} & 1.5 & - & 1.5 & - & 3.4 & - & & \\
\hline & $\mathrm{N}$ & $\%$ & $\mathrm{~N}$ & $\%$ & $\mathrm{~N}$ & $\%$ & & \\
\hline $\mathrm{ED}$ visit $^{\mathrm{e}}$ & 69 & 29 & 44 & 39 & 4 & 31 & 1.0 & 0.765 \\
\hline Readmission $^{\mathrm{e}}$ & 19 & 8 & 18 & 16 & 2 & 15 & 0.609 & 1.0 \\
\hline Reoperation $^{\mathrm{e}}$ & 11 & 5 & 10 & 9 & 0 & 0 & 1.0 & 0.388 \\
\hline
\end{tabular}

$O A R$ outpatient anorectal cases, $A v g$ average, $S D$ standard deviation, $L O S$ length of stay, $I C U$ intensive care unit, $E D$ emergency department

${ }^{a}$ The resource utilization outcomes (i.e. LOS, ICU, overall utilization and ICU utilization) of this group is equivalent to those of the historical overall since outpatient cases were excluded from the calculations of these variable

${ }^{\mathrm{b}}$ Comparison between historical overall and COVID groups

${ }^{\mathrm{c}}$ Comparison between historical minus OAR and COVID groups

${ }^{\mathrm{d}}$ Patient-days (and patient-ICU days) per week

${ }^{\mathrm{e}}$ Within 30 days

The baseline backlog of 73 patients corresponds with a baseline wait time of 9.6 weeks. If the number of weekly new case requests return to historical numbers ( 7.6 new cases per week), a new baseline wait time of 12.5 weeks would be established, marking a $23 \%$ increase in wait time. In this scenario, the operative volume could not increase to eliminate the

Table 3 Comparisons of postoperative morbidity overall and excluding outpatient anorectal cases

\begin{tabular}{|c|c|c|c|c|c|c|c|c|c|}
\hline & & \multicolumn{2}{|c|}{ Historical overall $(n=239)$} & \multicolumn{2}{|c|}{ Historical minus OAR $(n=112)$} & \multicolumn{2}{|c|}{$\operatorname{COVID}(n=13)$} & \multirow[t]{2}{*}{$P$ value $^{\mathrm{a}}$} & \multirow[t]{2}{*}{$P$ value $\mathrm{b}^{\mathrm{b}}$} \\
\hline & & $N$ & $\%$ & $\mathrm{~N}$ & $\%$ & $\mathrm{~N}$ & $\%$ & & \\
\hline \multirow[t]{2}{*}{ Overall complication } & None & 191 & $80 \%$ & 66 & $59 \%$ & 7 & $54 \%$ & 0.037 & 0.772 \\
\hline & Any & 48 & $20 \%$ & 46 & $41 \%$ & 6 & $46 \%$ & & \\
\hline \multirow[t]{5}{*}{ Per CD classification } & 1 & 17 & $7 \%$ & 16 & $14 \%$ & 1 & $8 \%$ & & \\
\hline & 2 & 17 & $7 \%$ & 17 & $15 \%$ & 2 & $15 \%$ & & \\
\hline & 3 & 11 & $5 \%$ & 10 & $9 \%$ & 2 & $15 \%$ & & \\
\hline & 4 & 3 & $1 \%$ & 3 & $3 \%$ & 1 & $8 \%$ & & \\
\hline & Total & 48 & $20 \%$ & 46 & $41 \%$ & 6 & $46 \%$ & $0.267^{\mathrm{c}}$ & $0.263^{\mathrm{c}}$ \\
\hline \multirow[t]{7}{*}{ Per morbidity type ${ }^{\mathrm{d}}$} & Ileus & 22 & $24 \%$ & 22 & $24 \%$ & 2 & $18 \%$ & 1.0 & 1.0 \\
\hline & SSI & 31 & $34 \%$ & 30 & $33 \%$ & 4 & $36 \%$ & 1.0 & 0.726 \\
\hline & UTI & 4 & $4 \%$ & 4 & $4 \%$ & 0 & $0 \%$ & 1.0 & 1.0 \\
\hline & AKI & 5 & $5 \%$ & 5 & $6 \%$ & 1 & $9 \%$ & 1.0 & 1.0 \\
\hline & Leak & 9 & $10 \%$ & 9 & $10 \%$ & 1 & $9 \%$ & 1.0 & 1.0 \\
\hline & DVT/PE & 2 & $2 \%$ & 2 & $2 \%$ & 2 & $18 \%$ & 0.056 & 0.058 \\
\hline & Others $^{\mathrm{e}}$ & 19 & $21 \%$ & 18 & $20 \%$ & 1 & $9 \%$ & 0.461 & 0.695 \\
\hline
\end{tabular}

$O A R$ outpatient anorectal cases, $C D$ Clavien-Dindo, SSI surgical site infection, $U T I$ urinary tract infection, $A K I$ acute kidney injury, $D V T / P E$ deep venous thrombosis/pulmonary embolism

${ }^{a}$ Comparison between historical overall and COVID groups

${ }^{\mathrm{b}}$ Comparison between historical minus OAR and COVID groups

${ }^{\mathrm{c}} P$ values across all Clavien-Dindo classes (1 to 4 )

${ }^{\mathrm{d}}$ The sum of individual complications is greater than the total number of patients with complications since some individuals had more than one complication

${ }^{\mathrm{e}}$ Including blood product transfusion, mesh infection, bacteremia, enterocutaneous fistula, pleural effusion, acute urinary retention, high ileostomy output, ostomy outlet obstruction, septic shock, medication-induced rash 
Table 4 Characteristics of patient backlog

\begin{tabular}{llcc}
\hline & & $\mathrm{N}$ & $\%$ \\
\hline Per disposition & Outpatient anorectal & 47 & $68 \%$ \\
& Requiring admission & 22 & $32 \%$ \\
& Total & 69 & $100 \%$ \\
Procedure type $^{\mathrm{a}}$ & Segmental colectomy & 4 & $18 \%$ \\
& Subtotal/total colectomy & 0 & - \\
& LAR & 3 & $14 \%$ \\
& APR & 1 & $5 \%$ \\
& Ostomy creation & 3 & $14 \%$ \\
& Ostomy reversal & 5 & $23 \%$ \\
& Others & \\
& Cancer/mass & 6 & $27 \%$ \\
& IBD & 3 & $14 \%$ \\
& Diverticulitis & 1 & $5 \%$ \\
& Ostomy related & 4 & $18 \%$ \\
& Others & & \\
& & 6 & $27 \%$ \\
& & 8 & $36 \%$ \\
\hline
\end{tabular}

$L A R$ low anterior resection, $A P R$ abdominoperineal resection, $I B D$ inflammatory bowel disease

${ }^{a}$ For cases requiring postoperative admission.

${ }^{\mathrm{b}}$ Including hernia repair, rectopexy, Altemeier procedure, rectovaginal fistula repair, advanced endoscopic techniques (i.e., TAMIS, endoscopic submucosal dissection).

${ }^{\mathrm{c}}$ Including rectovaginal fistula, colocutaneous fistula, rectal prolapse, anastomotic stricture.

excess backlog of patients since the operative capacity of the hospital would already be at maximal capacity.

Another possibility is that new case requests may be higher than historical numbers after the pandemic. This would continue to increase the case backlog until new case requests return to historical numbers. Assuming a new case request number of 12.5 cases per week, a 2-, 4-, and 6-week duration of this increased number of case requests would establish a new surgery wait time of 13.8 weeks ( $44 \%$ increase), 15.0 weeks (57\% increase), and 16.3 weeks ( $70 \%$ increase), respectively.

\section{Discussion}

The COVID-19 pandemic has dramatically restructured healthcare systems across the globe. ${ }^{7-9}$ In an effort to prepare for a potential influx of COVID-19 patients, all elective colorectal surgeries, except cancer cases, were postponed until further notice. This study looked at the implications of such decision-making. Two aspects of how this change impacts colorectal surgery are of interest as follows: first, how hospital resource utilization has differed since the postponement of elective cases, and second, what the future burden of backlogged cases may look like going forward.
Our study showed a marked reduction (74\%) in cases done, with only oncologic resections and urgent/emergent procedures proceeding. The decrease was particularly pronounced for anorectal procedures, which showed an $87 \%$ drop in total cases performed. For these postponed patients, it is unknown how many will progress, requiring an urgent or emergent intervention. We did not find any cases of this, but it is a real possibility if the backlog continues to grow.

Despite a significant decrease in the total number of cases performed during the COVID group timeframe, the number of urgent/emergent (non-elective) cases remained steady at 1.7 cases per week. This is in contrast to what was reported in Lombardy, Italy, where there was an $86 \%$ decrease in emergency surgery cases within 1 month after lockdown. ${ }^{10}$ They postulated that a combination of factors, including increased remote patient care by general practitioners and lockdowninduced dietary/social behavior changes, may have contributed. However, since the reported emergent cases were not stratified by subspecialty, the colorectal-specific emergent case number is unknown.

The hospital resource utilization results in this study were mixed. Interestingly, the overall inpatient resource utilization decreased by only $48 \%$ despite a marked reduction of weekly case volume by $74 \%$. This change in overall resource utilization was much less than expected and not proportional to the decrease in case volume. This discordance between case volume reduction and resource utilization is likely due to a greater number of patients in the COVID group with higher ASA classification and higher acuity issues who were treated in a non-elective context. Another contributing factor was longer average ICU, resulting in a $132 \%$ increase in ICU resource utilization in the COVID group. This is an important finding given the need to preserve ICU-level resources for potential COVID-19 patients. Given that none of the COVID group patients were diagnosed or treated for COVID-19 and that resource utilization did not decrease proportionately to the decrease in overall case volume, it is likely that such resource utilization was driven to a large degree by emergent cases. This finding may imply that making our operating rooms available to select elective cases will not significantly limit our ability to provide necessary care to COVID patients.

The differences in patient characteristics between the historical and COVID groups accurately reflect the changes experienced by the healthcare system during the COVID period. The greater proportion of patients with higher ASA classification in the COVID group is expected given that such patients are more likely to present in an urgent/emergent fashion. Despite the higher operative risk patients in the COVID group, the postoperative morbidity was similar between the two groups; however, the COVID group had a small sample size with insufficient power to see a difference and shorter follow-up period, which may result in incomplete capture of all morbidities. The procedure types were comparable, with 


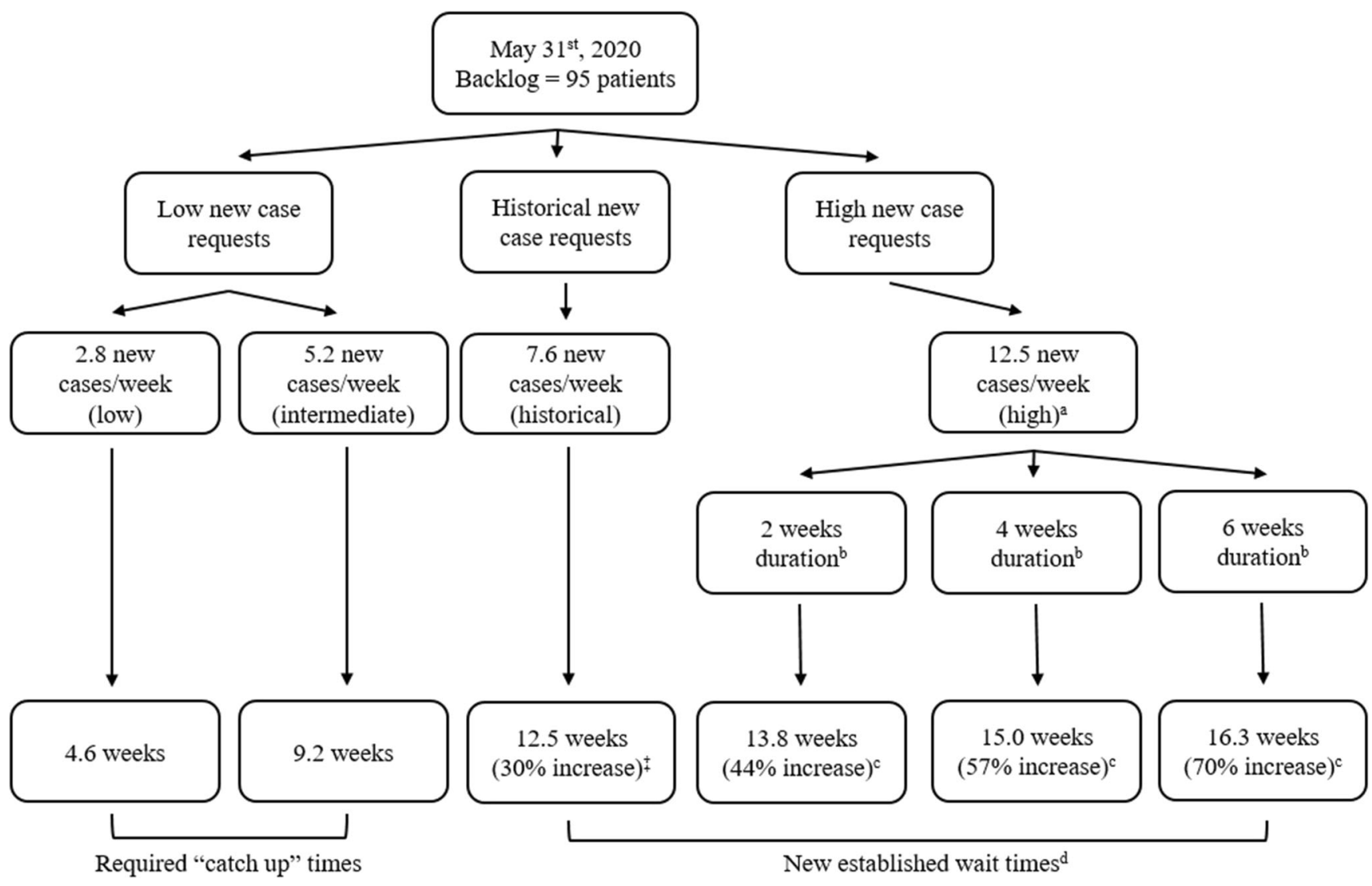

Fig. 1 A schematic outline of estimated catch up and new wait times. Based on various hypothetic (modeled) rates of new cases requests per week starting May 31, 2020. (a) new cases/week calculated by pandemic backlog case requests added to historical new case requests for possible

upstroke of new cases. (b) After backlog clearance, the new case requests drop back down to historical numbers (i.e., 7.6 new cases per week). (c) Relative increase from baseline wait time (see d). (d) Time between surgery scheduling and day of surgery.

the exception of outpatient anorectal cases, which were cancelled in the COVID group. The one anorectal procedure performed in the COVID group was an emergent case, performed for massive bleeding from a rectal Dieulafoy lesion. All elective cases in the COVID group were cancer cases, consistent with our mandated institutional guideline.

Though necessary at this time, the increasing backlog of patients poses significant future logistical challenges. Our resource-limited safety-net hospital have been running on full operative capacity historically and is unlikely to exceed our historical case volume even after the pandemic, especially in the current setting of case backlogs steadily accruing across all surgical services. There are two possibilities assuming the optimal scenario of immediate resumption of normal operative capacity after May 31st. In the best-case scenario, case request numbers are lower than historical numbers, which may be possible in the setting of potential patient unwillingness to come to the hospital, and "catch up" times range from 4.6 to 9.2 week. In the other, case requests surpass historical numbers due to either the progression of disease or the delay in seeking care during the COVID timeframe, establishing new wait times that are $23 \%$ to $70 \%$ longer than pre-COVID era. Even 1 month's duration of an increased number of new case requests could drive the surgery wait time to 15.0 weeks, a $57 \%$ increase from the historical baseline. Increased healthcare utilization and cost have been reported for colorectal cancer patients with long wait times, ${ }^{11}$ and a concern is that this may apply to benign diseases as well.

The acuity of patients as the pandemic resolves may also pose clinical challenges. If indeed there is a post-pandemic surge of surgical cases, ${ }^{10,12,13}$ despite continuing surgical care for colorectal cancer patients, there will be inevitable delays in diagnosis and treatment. This may result in an unprecedented increase in the number of new and advanced cancer patients in the post-pandemic period. ${ }^{12,}{ }^{14}$ Though the majority of non-cancer cases may tolerate treatment postponement without significant negative outcomes, there is concern for disease progression in select benign cases that may present in an urgent fashion. This includes recurrent diverticulitis episodes, incarceration/strangulation of parastomal hernias, Crohn's disease flares, and recurrent abscess in pilonidal disease and anal fistulas. In our backlog of cases, 25 patients (36\%) fall into this high-risk category. Furthermore, the direct effect of COVID-19 infections in colorectal patients awaiting 
treatment is unknown. This may have a significant impact on the hospital's ability to effectively deliver quality surgical care and strain an already overburdened county healthcare system.

In anticipation of these challenges, multiple scoring systems triaging surgical patients have been reported recently. ${ }^{15}$, ${ }^{16}$ These triaging methods attempt to balance the complexity of surgical patient care in this era by taking into account patient, surgical team, hospital resource, and COVID-19 transmission risk-related factors. Our institution plans to adopt the medically necessary time-sensitive (MeNTS) scoring system developed by Prachand et al. that involves 21 procedure, disease, and patient factors, with a 5-point scale for each factor. ${ }^{15}$ The score ranges from 21 to 105 , with higher scores associated with poorer outcomes and higher resource utilization. The upper threshold score for procedures deemed not justifiable in the current climate is determined by individual hospital systems based on dynamically changing local disease and resource use patterns. Additionally, the scoring system emphasizes availability of nonoperative therapies as opposed to stratifying risk by specific disease categories, allowing for broad applications across all surgical specialties. At the start of the pandemic, our institution implemented a policy of having each surgical case reviewed by the operating room medical director and the chief of anesthesia based on urgency. As the pandemic-related restrictions are lifted, the MeNTS scoring system will be utilized to systematically prioritize patients who have higher risk of failing nonoperative therapy.

The limitations of this study relate to its retrospective nature and limited follow-up data from the COVID group due to the short amount of time since the surgical policy was implemented. Surgical cases from 2018 were reviewed given the remote timing from the advent of the pandemic, though a review of cases from 2019 may have been a better approximation of typical case volume. Nonetheless, a 6-month period of 2018 was reviewed, compared with the 38 days of the COVID-19 group timeframe, to eliminate any monthly variation in baseline case volume. Furthermore, estimates of future surgical volume are speculative, and it remains unknown if pre-COVID surgical volumes will be obtainable, owing to requirements for testing, operating room sterilization and a variety of other logistical constraints. Furthermore, these estimates were based on the best-case scenario of resuming full operative capacity; the reality is likely a step-wise gradual increase in operative capacity, for which the exact timing and step-wise intervals are not known. We also did not examine the role of both diagnostic and therapeutic colonoscopy by our service, which was not performed during this period. In the limited number of patients for which we perform screening colonoscopies, we have a very low (less than 5\%) rate of identifying a need for surgery. Though there likely will be delays, the resumption of colonoscopies will inevitably affect the influx of new patients, many of whom may be diagnosed at more advanced stages of their disease process. Finally, this study evaluated the surgical volume at one county hospital, and the findings may not be generalizable to other institutions.

Understanding that our calculations rely on assumptions, we aim to offer a glimpse into what the future may look like post-COVID. While our predictions are just that, what is undeniable is that such an unprecedented disruption of the healthcare system will inevitably have ripple effects impacting all elements of not just the practice of colorectal surgery but of all of medicine. At best, the end result will be increased wait times, but the unfortunate reality is that many unforeseen consequences likely lie ahead.

\section{Conclusion}

The effects of dramatically restructuring a healthcare system in preparation for the COVID-19 pandemic at our institution resulted in not only a decrease in overall hospital resource utilization but also an increase in ICU utilization for colorectal surgery patients. The backlog of cancelled elective colorectal cases is expected to increase and poses significant logistical and clinical challenges in the post-pandemic period. Surgical triage systems, as well as the implementation of preoperative screening protocols for COVID-19, will play critical roles in strategically and effectively managing the inevitable backlog of surgical patients as the COVID-19 pandemic continues.

Acknowledgments We would like to acknowledge Ziyad Jabaji, MD, Jason Chen, MD, Leo Granados, PA-C, and Lydia Ruiz, RN, in their unwavering support and flexibility during this tumultuous time.

Authors' Contributions All authors contributed appropriately according to the Definition of Authorship per the International Committee of Medical Journal Editors (ICMJE).

\section{Compliance with Ethical Standards}

Conflict of Interest All authors have no relevant conflicts of interest to disclose.

\section{References}

1. Gorbalenya AE, Baker SC, Baric RS, Groot RJ, Drosten C, Gulyaeva AA, Haagmans BL, Lauber C, Leontovich AM, Neuman BW, Penzar D, Perlman S, Poon LLM, Samborskiy D, Sidorov IA, Sola I, Ziebuhr J. Severe acute respiratory syndromerelated coronavirus: The species and its viruses - a statement of the Coronavirus Study Group. bioRxiv. 2020; https://doi.org/10.1101/ 2020.02.07.937862

2. World Health Organization. Naming the coronavirus disease (COVID-19) and the virus that causes it. World Health Organization website. https://www.who.int/emergencies/diseases/ novel-coronavirus-2019/technical-guidance/naming-thecoronavirus-disease-(covid-2019)-and-the-virus-that-causes-it. Accessed April 28, 2020. 
3. World Health Organization. Novel Coronavirus (2019-nCoV) Situation Report - 11. World Health Organization website. https:// www.who.int/docs/default-source/coronaviruse/situation-reports/ 20200131-sitrep-11-ncov.pdf?sfvrsn = de7c0f7_4. Jan 31, 2020. Accessed April 28, 2020.

4. World Health Organization. WHO Timeline - COVID-19. World Health Organization website. https:/www.who.int/news-room/ detail/27-04-2020-who-timeline\%2D\%2D-covid-19. April 27, 2020. Accessed April 28, 2020.

5. American College of Surgeons. COVID-19: Recommendations for Management of Elective Surgical Procedures. American College of Surgeons website. https:/www.facs.org/covid-19/clinicalguidance/elective-surgery. March 13, 2020. Accessed April 28, 2020.

6. Centers for Disease Control and Prevention. Healthcare Facilities: Preparing for Community Transmission. Centers for Disease Control and Prevention website. https://www.cdc.gov/ coronavirus/2019-ncov/hcp/guidance-hcf.html?CDC_AA_refVal, https://www.cdc.gov/coronavirus/2019-ncov/healthcare-facilities/ guidance-hcf.html. February 29, 2020. Accessed April 28, 2020.

7. Mcbride KE, Brown KGM, Fisher OM, Steffens D, Yeo DA, Koh CE. Impact of the COVID-19 pandemic on surgical services: early experiences at a nominated COVID-19 centre. ANZ Journal of Surgery. doi:https://doi.org/10.1111/ans. 15900

8. Di Saverio, S., Pata, F., Gallo, G., Carrano, F., Scorza, A., Sileri, P., Smart, N., Spinelli, A. and Pellino, G. (2020), Coronavirus pandemic and Colorectal surgery: practical advice based on the Italian experience. Colorectal Dis. Accepted Author Manuscript. doi:https://doi.org/10.1111/codi.15056

9. COVIDSurg Collaborative. Global guidance for surgical care during the COVID-19 pandemic [published online ahead of print on Apr 15 2020]. Br J Surg. 2020; doi:https://doi.org/10.1002/bjs. 11646

10. Patriti A, Eugeni E, Guerra F. What happened to surgical emergencies in the era of COVID-19 outbreak? Considerations of surgeons working in an Italian COVID-19 red zone [published online ahead of print, 2020 Apr 23]. Updates Surg. 2020;1-2. doi:https://doi.org/ 10.1007/s13304-020-00779-6

11. Delisle M, Helewa RM, Ward MAR, Hochman DJ, Park J, Mckay A. The Association Between Wait Times for Colorectal Cancer Treatment and Health Care Costs: A Population-Based Analysis. Dis Colon Rectum. 2020;63(2):160-171. doi:https://doi.org/10. 1097/DCR.0000000000001517

12. Spinelli A and Pellino G. COVID-19 pandemic: perspectives on an unfolding crisis. Br J Surg. 2020; doi:https://doi.org/10.1002/bjs. 11627

13. Sanz-Santos J, Rami-Porta R, Call S. Lessons already learnt from the Covid-19 pandemic [published online ahead of print, 2020 Apr 22]. J Thorac Oncol. 2020;S1556-0864(20)30326-9. doi: https://doi.org/10.1016/j.jtho.2020.04.012

14. Pellino G, Spinelli A. How COVID-19 Outbreak Is Impacting Colorectal Cancer Patients in Italy: A Long Shadow Beyond Infection. Dis Colon Rectum. 2020;63(6)720-722. doi:https://doi. org/10.1097/DCR.0000000000001685.

15. Prachand VN, Milner R, Angelos P, Posner MC, Fung JJ, Agrawal N, Jeevanandam V, Matthews JB. Medically Necessary, TimeSensitive Procedures: Scoring System to Ethically and Efficiently Manage Resource Scarcity and Provider Risk During the COVID19 Pandemic [published online ahead of print, 2020 Apr 9]. J Am Coll Surg. 2020;S1072-7515(20)30317-3. doi:https://doi.org/10. 1016/j.jamcollsurg.2020.04.011

16. Carlson GL. Prioritizing Access to Surgical Care During the Coronavirus Pandemic [published online ahead of print on April 2 2020]. Dis Colon Rectum. 2020; doi:https://doi.org/10.1097/ DCR.0000000000001698

Publisher's Note Springer Nature remains neutral with regard to jurisdictional claims in published maps and institutional affiliations. 